\title{
Erratum: Unifying renormalization group and the continuous wavelet transform [Phys. Rev. D 93, 105043 (2016)]
}

\author{
M. V. Altaisky॰ \\ (A) (Received 12 February 2022; published 28 February 2022)
}

DOI: 10.1103/PhysRevD.105.049901

This erratum addresses misprints in the paper "Unifying renormalization group and the continuous wavelet transform" by M. V. Altaisky published in Phys. Rev. D 93, 105043 (2016).

On page 4 of this paper misprints have been found in the right-hand side of Eq. (18). The value of parameter $\alpha$ is different for Eqs. (18) and (19); in Eq. (18) $\alpha=A s$, not $\alpha=A m$ as in Eq. (19). The correct form of the system of these two equations should be

$$
\begin{gathered}
\frac{\partial \lambda}{\partial \mu}=3 \lambda^{2} \alpha^{2} \frac{\partial X_{1}^{4}}{\partial \alpha^{2}}=\frac{3 \lambda^{2}}{16 \pi^{2}} \frac{1-e^{-\alpha^{2}}}{\alpha^{2}} e^{-\alpha^{2}}, \quad \text { where } \alpha=A s \\
\frac{1}{m^{2}} \frac{\partial m^{2}}{\partial \mu}=\frac{\lambda}{32 \pi^{2} \alpha^{2}}-\frac{\lambda}{16 \pi^{2}}+\frac{\lambda}{16 \pi^{2}} 2 \alpha^{2} e^{2 \alpha^{2}} \operatorname{Ei}_{1}\left(2 \alpha^{2}\right),
\end{gathered}
$$

where $\alpha=A m$. 